\title{
The impact of different preanalytical methods related to CA 15-3 determination in frozen human blood samples: a systematic review
}

\author{
Luigi Coppola, Alessandra Cianflone, Katia Pane, Monica Franzese, Peppino Mirabelli ${ }^{*}$ (D) and Marco Salvatore
}

\begin{abstract}
Background: The determination of CA 15-3 is useful for monitoring breast cancer patients. Several retrospective studies determined CA 15-3 levels in frozen samples to evaluate the sensitivity and specificity of novel biomarkers in relation to breast cancer; however, freeze-thaw cycles, as well as preanalytical variables before sample storage, are not always reported. Here, we analyzed the current scientific literature to identify possible critical aspects related to CA 15-3 determination in frozen-stored human serum/plasma samples.

Methods: We obtained data from 4 different bibliographic databases: Web of Science, Embase, PubMed, and Cochrane Library. We followed the PRISMA guidelines to screen and select the eligible articles discussed in the final revision.

Results: Initially, 674 scientific papers were evaluated, and after the application of the screening and eligibility criteria, 18 studies were included in the qualitative synthesis. The analysis reported an important level of heterogeneity concerning the preanalytical phase before sample storage.

Conclusion: Although advances in healthcare have been achieved using certified workflows in medical diagnostics, standardized preanalytical processes are not always applied when referring to frozen-stored biosamples. Biobanks will guarantee the best possible conditions for the storage of human biological samples to be used in clinical research. The use of certified bioresources will favor the optimal development and introduction of new disease biomarkers.
\end{abstract}

Keywords: Frozen human blood, CA 15-3 determination, Preanalytical phase, Tumor biomarkers, Biochemical indicators

\section{Background}

Biomarkers are biomolecules that usually serve as indicators of pathological processes or for having clinical information about pharmacological responses to drug treatment [1]. Due to their clinical usefulness, biomarker discovery and validation represent one of the pillar strategies for research in the field of personalized medicine [2] for better diagnosis and prognosis [3, 4]. However, it

\footnotetext{
* Correspondence: peppino.mirabelli@synlab.it;

direzionescientifica.irccssdn@synlab.it

IRCCS SDN, Via E. Gianturco, 80143 Naples, Italy
}

is important to consider that the life-cycle stages of biosamples, i.e., collection, accession, acquisition, identification, preservation, long-term storage, quality control (QC), transport, and sharing of biomaterials, represent a major source of heterogeneity among biorepositories. Consequently, the use of frozen biosamples, without the application of standardized storage procedures, can negatively affect biomarker identification and validation phases [5] as well as result comparisons across studies.

In this context, to better understand the critical aspects related to the management of human biological

C C The Author(s). 2021 Open Access This article is licensed under a Creative Commons Attribution 4.0 International License, which permits use, sharing, adaptation, distribution and reproduction in any medium or format, as long as you give appropriate credit to the original author(s) and the source, provide a link to the Creative Commons licence, and indicate if changes were made. The images or other third party material in this article are included in the article's Creative Commons licence, unless indicated otherwise in a credit line to the material. If material is not included in the article's Creative Commons licence and your intended use is not permitted by statutory regulation or exceeds the permitted use, you will need to obtain permission directly from the copyright holder. To view a copy of this licence, visit http://creativecommons.org/licenses/by/4.0/. The Creative Commons Public Domain Dedication waiver (http://creativecommons.org/publicdomain/zero/1.0/) applies to the data made available in this article, unless otherwise stated in a credit line to the data. 
samples, we decided to evaluate how the preanalytical variables and storage conditions (including freeze-thaw cycles) are correctly reported in the literature for CA 153 , a commonly used biomarker for monitoring breast cancer $(\mathrm{BC})$. We selected this marker since it is widely used in clinical practice for patient stratification in retrospective case-control studies as well as for performance comparison with novel introduced biomarkers. In doing so, we reviewed the literature of the past 10 years on different scientific databases (PubMed, Embase, Web of Science, and Cochrane Library), focusing our attention on technical approaches (frozen or fresh samples, storage temperature, type of biological samples used, cutoff adopted, etc.) and selecting the CA 15-3 determinations obtained from thawed samples. Generally, CA 15-3 represents the soluble form of mucin-1 (MUC-1) antigen. This surface protein is upregulated (10-fold higher than in adjacent normal glandular epithelium) on the surface of breast cancer cells [6], and after being shed from the $\mathrm{BC}$ cell surface, it is released into the bloodstream and used as a disease biomarker, as stated by different scientific studies $[7,8]$. Despite its association with $\mathrm{BC}$ cells, an increase in CA 15-3 can be detected in some benign conditions, such as liver disease and benign breast, lung, or ovarian disease [9]; therefore, it is not considered a specific BC biomarker for diagnostic purposes.

However, the European Group on Tumor Markers (EGTM), in agreement with the National Academy of Clinical Biochemistry (NACB) guidelines, suggests that increasing levels of serum tumor markers may often precede disease recurrence $[10,11]$. Thus, CA 15-3 levels, according to the EGTM in agreement with the NACB guidelines, serve mainly for monitoring $\mathrm{BC}$ patients at risk of developing metastatic disease since increasing levels of CA 15-3 may often precede disease recurrence $[10,11]$.

Technically, the CA 15-3 value is determined in fresh blood serum samples; however, in biomedical research, the determination of biomarkers from thawed human biological samples is frequently carried out to evaluate a new analytical technique or for comparison with other biomarkers. In this regard, several studies systematically investigated the effect of storage conditions on blood samples for the measurement of different analytes [1214]. While CA 15-3 preanalytical variables have already been studied in previous works [15-17], the preanalytical factors influencing CA 15-3 determination in frozen human blood samples have never been studied in detail. For this reason, we performed a critical review of literature studies on CA 15-3 determination in frozen-thawed samples. Here, we emphasize study characteristics based on preanalytical variables and discuss the results in light of standard procedures for the proper biobanking and handling of human biosamples [5]. We believe that correct storage of biological samples is necessary not only for retrospective CA 15-3 determination but also for the identification and validation of novel clinical biomarkers. In this way, scientific results will be more comparable across studies, especially for the introduction of novel biomarkers.

\section{Materials and methods}

The paper was prepared based on the Preferred Reporting Items for Systematic Reviews and Meta-Analyses (PRISMA) standards and guidelines (Additional file 1) [18]. All data originate from previously published experiments in international peer-reviewed journals.

\section{Search strategy and eligibility criteria}

For eligibility criteria on study characteristics, we included English peer-reviewed papers involving CA 15-3 in human fluids such as serum, plasma, or whole blood and excluded in vitro studies. Only original articles with publication status information within a time interval of 10 years (January 2010 up to April 2020) were included, while case reports, reviews, and editorials were excluded. All studies were identified by searching the PubMed, Embase, Web of Science, and Cochrane Library databases by using the following search keywords: "ca 15-3," "ca 15.3," "muc1," "mucin 1," "carbohydrate antigen 153," "carbohydrate antigen 15.3," "serum," "plasma," and "blood" with the last search date on 1 April 2020.

\section{Data extraction and collection}

Two authors carried out article searches and data collection independently (L.C. and A.C.). A third reviewer (K.P.) independently carried out data extraction and reviewed the selected published articles to confirm that they met the inclusion criteria. The extraction of data for the following predetermined variables was performed: study design, study period, the outcome of the study, and the presence of follow-up in time-to-event studies, i.e., follow-up length, age, patient subgroups, age, and sex-matched case-control, association with clinical-pathological features, statistical methods for CA 15-3 determination, and biospecimen source as described below in the data item section. Any disagreements that arose between the reviewers were resolved through discussion with a fourth, fifth, or sixth reviewer (P.M., M.F., and M.S.).

\section{Study selection}

Circulating tumor biomarker detection has clinical utility for patient management and is determined using fresh serum or plasma samples [19]. Conversely, frozen samples are required for retrospective analysis focused on the identification or validation of novel biomarkers as well as the establishment of new 
analytical technologies. For this systematic review, we included studies on frozen-thawed samples according to the PRISMA flow diagram shown in Fig. 1. Using four different databases (PubMed, Embase, Web of Science, and Cochrane Library), we selected papers with retrospective, prospective, or cross-sectional studies, resulting in a total of 674 papers. Initial screening of the titles led to the exclusion of 285 articles, while title and abstract screening led to the exclusion of 46 articles. Among the 70 full-text articles assessed for eligibility, we excluded 52 full-text articles since the CA 15-3 determinations were not carried out on thawed human biological samples, and we excluded 1 full-text article because the sample storage temperature was unknown [20]. Finally, we included 18 full-text articles for the final review; Excel (Microsoft Office 2019, software) was used to remove duplicate articles.

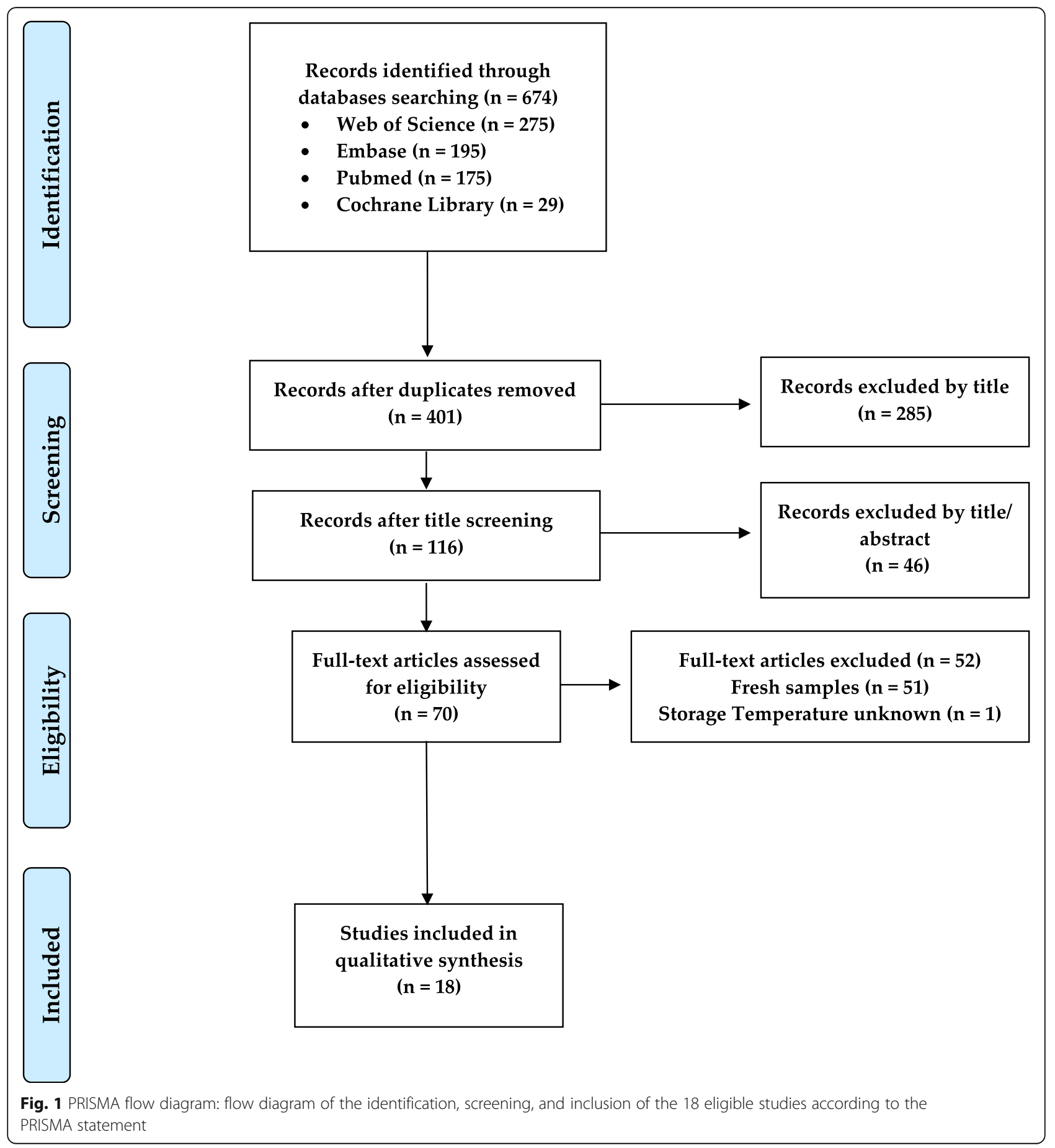




\section{Data items}

Data items for searching papers were specifically related to the presence of the following variables of interest: biospecimen type, i.e., human sample type, fresh or frozen human samples, and sample preanalytical variables, such as (i) the handling of aliquots of the total biospecimen; (ii) freeze-thaw cycle and temperature; (iii) aliquot centrifugation before testing; and (iv) interim aliquot storage until analysis. Searching for CA 15-3 determination on frozen-thawed samples, we assumed that fresh samples could also be included in the selected papers since both sample types can be assayed concomitantly. Since CA $15-3$ is not a specific $\mathrm{BC}$ biomarker, we simplified the disease and/or clinical outcome search to cover more literature findings. Thus, different diseases and outcomes (disease monitoring, recurrence, or follow-up) are included in the final selected studies.

\section{Summary measures}

We assumed as principal summary measure the CA 153 cutoff value as the difference in mean concentrations between the case groups and the control groups with the standard mean error or standard deviation for statistical significance as reported in each study.

\section{Risk of bias}

We carried out the risk of bias assessment by objectively evaluating the selected publications with the following questions: (1) Can we find confounding factors for sample handling? (2) Can we reproduce the sample freezethaw cycle? (3) Are we confident in the appropriate balance of the sample sizes of the case and control groups? We assessed the risk of bias across studies by using the Newcastle-Ottawa Scale (NOS) for case-control study quality assessment [21].

\section{Quality assessment of selected studies}

We carried out a quality assessment using the NewcastleOttawa Scale (NOS) for the case-control studies (Additional file 2) [21]. We judged study group comparability items (maximum two stars for a single item) based on arbitrarily important factors, such as one star to a study that explained the freeze-thaw cycle, such as (i) the handling of aliquots of the total biospecimen; (ii) freeze-thaw cycles and temperatures; (iii) aliquot centrifugation before testing; and (iv) interim aliquot storage until analysis, and one star to a study that had a balanced case-control study sample size. The sample size balance estimation is arbitrarily defined using the threshold of $n \leq 10$ samples for group comparison.

\section{Results}

\section{Study characteristics}

The final included articles $(n=18)$ encompassed casecontrol studies based on 17 retrospective study designs [22-38] and one prospective study [39]. The sample size across studies ranged from a minimum of 30 [39] to a maximum of 472 [27]. Table 1 reports a summary of the findings of the 18 papers selected for the critical review classified according to study type, the number of studies, biospecimen type, storage temperature $\left({ }^{\circ} \mathrm{C}\right)$, patient status/disease, measurement unit, and the kit or instrument used. Specifically, according to the biospecimen type used for CA 15-3 determination in the 18 included papers, 14 studies (approximately $80 \%$ ) used serum samples [22-32, 34, 35, 39], while the remaining 4 were structured as follows: (i) Zajkowska et al. used exclusively plasma samples [33], (ii) Christenson et al. focused on methodological comparison and assayed CA 15-3 in both serum and plasma samples [36], (iii) Saba et al. measured CA 15-3 in both serum and pleural effusion [37]; and (iv) Laidi et al. determined CA 15-3 in serum and saliva biosamples [38]. Regarding the preanalytical variable of storage temperature, 9 studies out of the 18 (50\%) $[26-30,32,34,35]$ stored biosamples at $-80^{\circ} \mathrm{C}$ until analysis, 6 studies stored biosamples at $-20^{\circ} \mathrm{C}$ $[22-25,37,39]$, one study [31] stored biosamples at $70{ }^{\circ} \mathrm{C}$, one study stored plasma at $-85^{\circ} \mathrm{C}$ [33], and only Christenson et al. assayed CA 15-3 from biosamples stored at both $-20^{\circ} \mathrm{C}$ and $-70{ }^{\circ} \mathrm{C}[36]$.

In the selected papers, the CA 15-3 measurement was mainly (approximately 80\%) evaluated for monitoring $\mathrm{BC}$; in fact, 14 out of the 18 articles were focused on this pathology [22-29, 31-35, 38]. Interestingly, 4 articles underlined that CA 15-3 determination is also executed for other needs; indeed, this tumor marker was even analyzed in adnexal mass patients and in patients with pleural effusion [30, 37]. Additionally, the accuracy of CA 15-3 determination was also evaluated in 30 pregnant women, and in one case, it was evaluated in an instrument comparison study for measuring multiple disease biomarkers from BC patient samples [36, 39].

We also studied other variables, such as information related to the kits or instruments used and cutoff values. In 15 of the 18 studies, the measurement unit for CA 15-3 was the difference in CA 15-3 mean concentration between the case groups and the control groups expressed in $\mathrm{U} / \mathrm{mL}$ with the standard mean error or standard deviation for statistical significance; in Saba et al. [37] and Tang et al. [35], ng/mL was reported; and in Metwally et al. [31], U/L was reported. To determine CA 15-3, 4 selected studies used chemiluminescent enzyme immunoassays (CLEIAs) [27, 28, 34, 35]; 3 studies used enzyme-linked immunosorbent assays (ELISAs) $[22,37,38]$; and in other studies, enzyme immunoassays 
Table 1 Summary of the findings of the 18 eligible articles on CA 15-3 determination from thawed human biosamples

\begin{tabular}{|c|c|}
\hline & $\begin{array}{l}\text { No. of studies } \\
\text { [reference] }\end{array}$ \\
\hline \multicolumn{2}{|l|}{ Study type } \\
\hline Retrospective study & 17 [22-38] \\
\hline Prospective study & $1[39]$ \\
\hline \multicolumn{2}{|l|}{ Biospecimen type } \\
\hline Serum & $14[22-32,34,35,39]$ \\
\hline Plasma & $1[33]$ \\
\hline Serum and plasma & $1[36]$ \\
\hline Serum and pleural effusion & $1[37]$ \\
\hline Serum and saliva & $1[38]$ \\
\hline \multicolumn{2}{|l|}{ Storage temperature $\left({ }^{\circ} \mathrm{C}\right)$} \\
\hline-80 & $9[26-30,32,34,35,38]$ \\
\hline-20 & $6[22-25,37,39]$ \\
\hline-70 & $1[31]$ \\
\hline-85 & $1[33]$ \\
\hline$-20 ;-70$ & $1[36]$ \\
\hline \multicolumn{2}{|l|}{ Patient status/disease } \\
\hline Breast cancer & $14[22-29,31-35,38]$ \\
\hline Pleural effusion & $1[37]$ \\
\hline Adnexal masses and ovarian cancer & $1[30]$ \\
\hline Pregnancy & $1[39]$ \\
\hline Multiple disease & $1[36]$ \\
\hline \multicolumn{2}{|l|}{ Measurement unit } \\
\hline $\mathrm{U} / \mathrm{mL}$ & $\begin{array}{l}15[22-30,32-34,36,38, \\
39]\end{array}$ \\
\hline $\mathrm{ng} / \mathrm{mL}$ & $2[35,37]$ \\
\hline$U / L$ & $1[31]$ \\
\hline \multicolumn{2}{|l|}{ Kit or instrument } \\
\hline CLEIA & $4[27,28,34,35]$ \\
\hline EIA & $3[25,29,39]$ \\
\hline ELISA & $3[22,37,38]$ \\
\hline $\begin{array}{l}\text { HumaReader Plus made by HUMAN } \\
\text { GmbH }\end{array}$ & $2[23,24]$ \\
\hline MEIA & $2[30,31]$ \\
\hline RIA & $2[26,32]$ \\
\hline CMIA & $1[33])$ \\
\hline Vista vs Advia Centaur & $1[36]$ \\
\hline
\end{tabular}

(EIAs) [25, 29, 39], microparticle enzyme immunoassays (MEIAs) [30, 31], radioimmunoassays (RIAs) [26, 32], and chemiluminescent microparticle immunoassays (CMIAs) [33] were used. Finally, 3 out of the 18 papers reported the semiautomated or automated instrument used [23, 24, 36]. Additionally, it is known that interim storage at low temperatures can affect the quality of human biological samples [40, 41]; unfortunately, only 1
(36) out of the 18 selected papers reported the freezing period before CA 15-3 determination.

\section{Synthesis of results}

Overall, the data in Table 1 emphasize that CA 15-3 determination is heterogeneous and is performed through different technical approaches. Critical differences were found in relation to the storage temperature, measurement unit, and kit or instrument used for testing new possible approaches for translational research. Additionally, it is necessary to emphasize other missing information, such as (i) the hemolysis of biological samples, (ii) the frozen storage duration from all papers except that of Christenson et al. [36], and (iii) how frozen samples were thawed before CA 15-3 determination. A more detailed description of the characteristics of the studies within the 18 selected articles is reported in Table 2.

\section{Risk of bias across studies}

We assessed the risk of bias across studies by evaluating the bias in the selection of cases and controls, as well as the comparability across studies for important factors such as the freeze-thaw cycle and handling to allow the reproducibility of the research and expose bias (Additional file 2). Cases in most studies were based on selfmedical reports and/or hospital examinations without multiple independent medical validations. Indeed, the consensus diagnosis performed by three specialists is described only in the paper by Sen et al. on ovarian cancer and adnexal mass investigation [30]. Most case-control studies are based on consecutive sampling from hospitals or universities/hospitals. This is an important bias that is generally present in the majority of the studies based on hospital-community controls rather than those based on population-based controls. In this context, confounding factors may arise because most of the "healthy subject" controls are referred to as "healthy volunteers" without further description. Indeed, 6 out of 18 articles clearly stated that controls had no history of the disease (endpoint), and only Said et al. described a full history of the interview for all cases and controls [24]. A risk of bias across the studies within all eligible articles was found when comparing the freeze-thaw cycle and handling of samples. Among the 18 eligible articles, that of Christenson et al. [36] described most of the preanalytical variables considered in this systematic review. Indeed, they reported sera and plasma handling, storage stability at different temperatures (Table 1), the origin of fresh-frozen samples from the commercial blood bank, and sample thawing for CA 15-3 determination at day 0 and after 1 year of storage.

Heterogeneity in the case ascertainment of exposure (no surgical record linkage) was found. Additionally, the case-control nonresponse rate was described in only one 
study [36]. Several studies focused on CA 15-3 determination for $\mathrm{BC}$ monitoring, such as disease recurrence or treatment follow-up. We found that Pedersen et al. [28] studied the risk of bias in sample handling since serum HER2 determination was carried out prospectively (on fresh samples), while serum CEA and CA 15-3 were analyzed retrospectively in samples stored at $80^{\circ} \mathrm{C}$. Additionally, the period of case follow-up was not reported in detail. Furthermore, Chukwurah et al. carried out BC surveillance in Nigerian women. Their CA 15-3 levels may be difficult to compare to those of other studies during the follow-up period (3 to 6 months, probably due to the Nigerian program's screening policy) [22]. Additionally, a risk of bias was present in the Svobodova et al. follow-up case-control study, whose case monitoring follow-up after surgery was carried out in different centers for longer intervals [27].

We found selection bias in some eligible studies that did not explain whether eligible patients were enrolled consecutively or randomly (Additional file 2).

\section{Discussion}

The present work aimed to evaluate the impact of preanalytical conditions related to CA 15-3 determination in frozen human samples and to highlight the importance of using standardized procedures across studies for comparing results across studies. The selected papers encompass 18 case-control studies focused on CA 15-3 determination in frozen serum or plasma samples. Most of these studies aimed to evaluate the sensitivity and specificity of novel circulating biomarkers in comparison to CA 15-3 in relation to $\mathrm{BC}$ or additional diagnostic applications of CA 15-3 apart from BC. The bibliometric analysis performed in this study revealed that several technical data related to the processing, storage period, and thawing conditions were missing or only poorly reported. Overall, the data in Table 1 emphasize that CA 15-3 determination was heterogeneous and was performed through different technical approaches. Critical differences were found in relationship to the storage temperature, measurement unit, and kit or instrument used for testing new possible approaches for translational research. Additionally, it is necessary to emphasize other missing information, such as (i) the hemolysis of biological samples, (ii) the frozen storage duration from all papers except that of Christenson et al. [36], and (iii) how frozen samples were thawed before CA 15-3 determination. These variables led to heterogeneous experimental conditions for CA 15-3 determination in the thawed samples across the included studies. Among the 18 eligible articles, only Christenson et al. [36] described that frozen samples $\left(-70^{\circ} \mathrm{C}\right)$ were tested within 1 year from the date of collection, and samples were thawed at room temperature for $30 \mathrm{~min}$ and recentrifuged at $1500 \times g$ for 2-5 min [36]. Although such reporting might seem "ancillary," it is important to adopt common collection, processing, storage, and thawing procedures for the management of biological samples for data reproducibility within the international scientific community. In this context, samples from biobanks might also guarantee higher-quality biosamples [42, 43] by minimizing the effect of preanalytical variables on the sample life cycle, including storage for a long period. One study out of the 18 selected papers assayed CA 15-3 in biological samples obtained from a biobank, i.e., Zaleski et al. from the Biofluid Biobank of the University Hospital Bonn [34]. In this case, it is important to highlight that obtaining samples from a biobank is optimal. Indeed, a biobank processes its samples following a standardized procedure [44]. Moreover, the same sample can be used by different researchers to produce comparable results and validate different markers in the same patient cohort.

Regarding the limitations of our study, we focused on 10 years (January 2010-April 2020) of literature studies for CA 15-3 determination and preanalytical variables affecting frozen biosample handling to evaluate a manageable number of articles and the most recent articles; however, we are aware that preanalytical variables affect not only frozen human samples but also likely every type of biosample.

\section{Conclusions}

One frontier of precision medicine is to identify noninvasive novel tumor biomarkers; consequently, the proper use of frozen-stored biosamples is of utmost importance. Here, we highlighted that heterogeneous preanalytical variables and storage conditions are applied when retrospective case-control studies are performed. This highlights an important source of variability across studies, which leads to results not always being comparable or reproducible. The use of standardized procedures for sample storage as well as the use of samples stored in biobanks should be promoted to obtain high-quality samples for biomarker discovery.

\footnotetext{
Abbreviations

ASCO: American Society of Clinical Oncology; BC: Breast cancer; CA 153: Cancer antigen 15-3; CLEIA: Chemiluminescent enzyme immunoassay; CMIA: Chemiluminescent microparticle immunoassay; EGTM: European

Group on Tumor Markers; EIA: Enzyme immunoassay; ELISA: Enzyme-linked immunosorbent assay; ESMO: European Society for Medical Oncology; MEIA: Microparticle enzyme immunoassay; NACB: National Academy of Clinical Biochemistry; NCCN: National Comprehensive Cancer Network; NOS: Newcastle-Ottawa scale; PRISMA: Preferred Reporting Items for Systematic Reviews and Meta-Analyses; QC: Quality control; RIA: Radioimmunoassay
} 


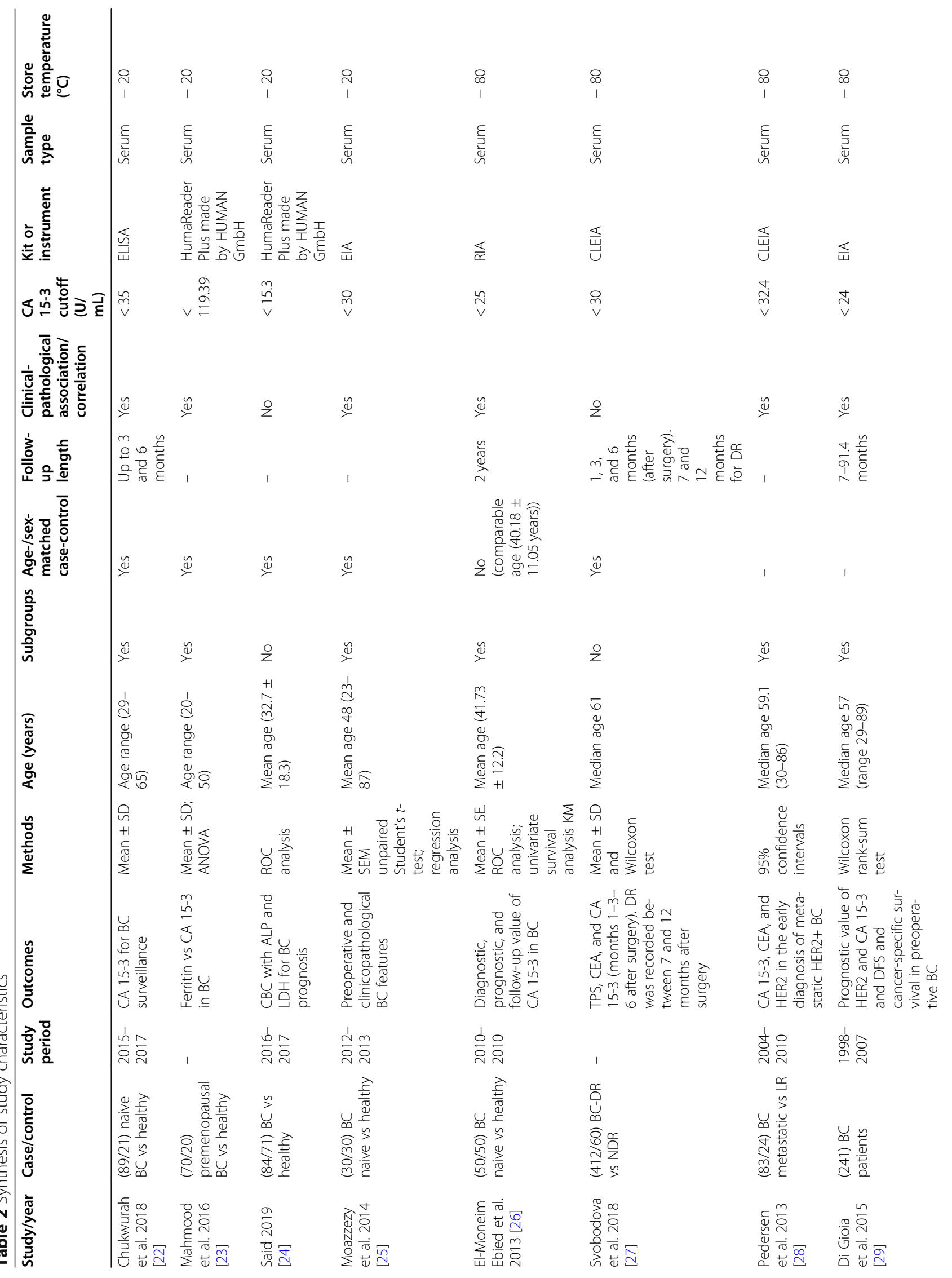




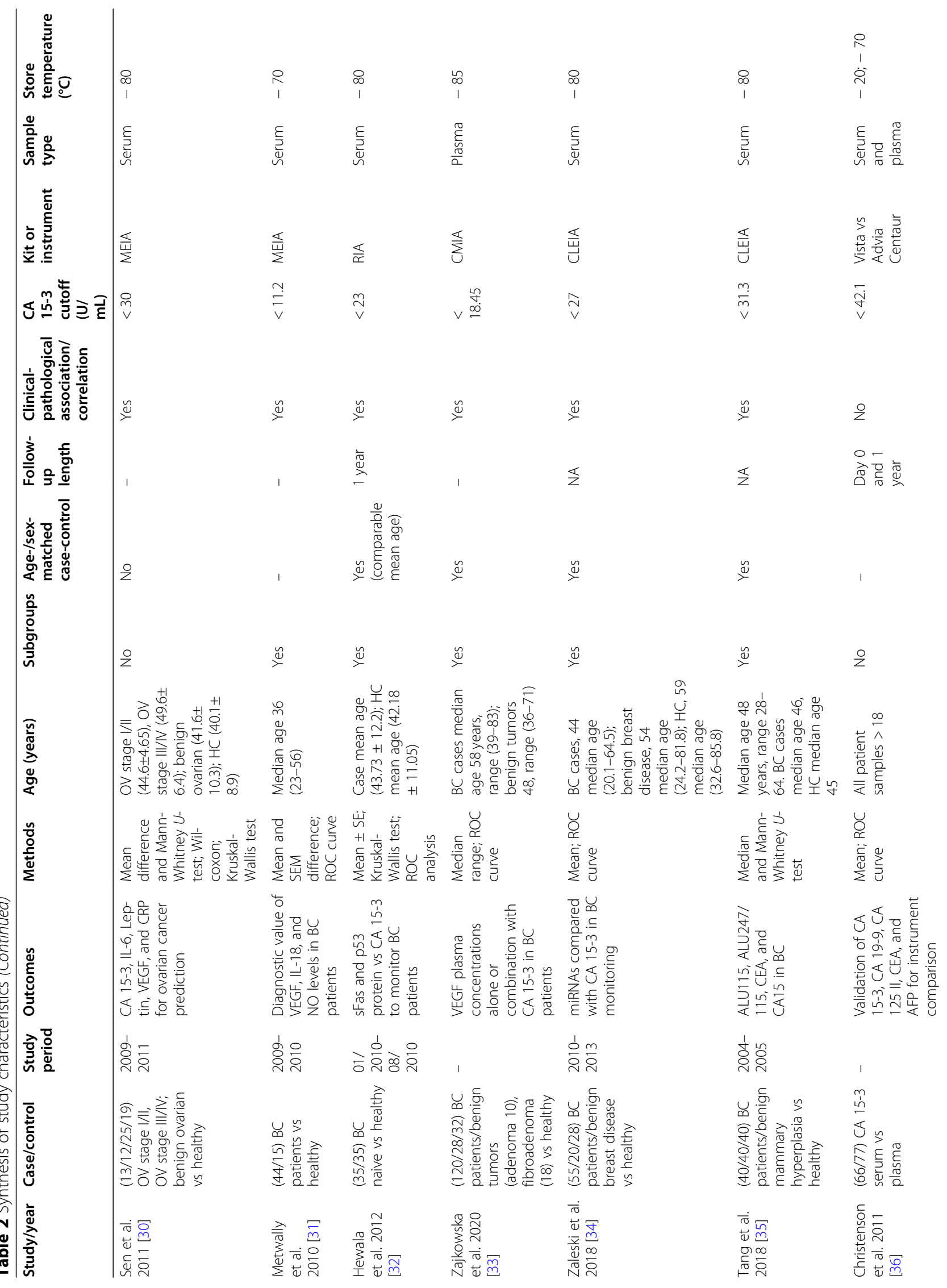




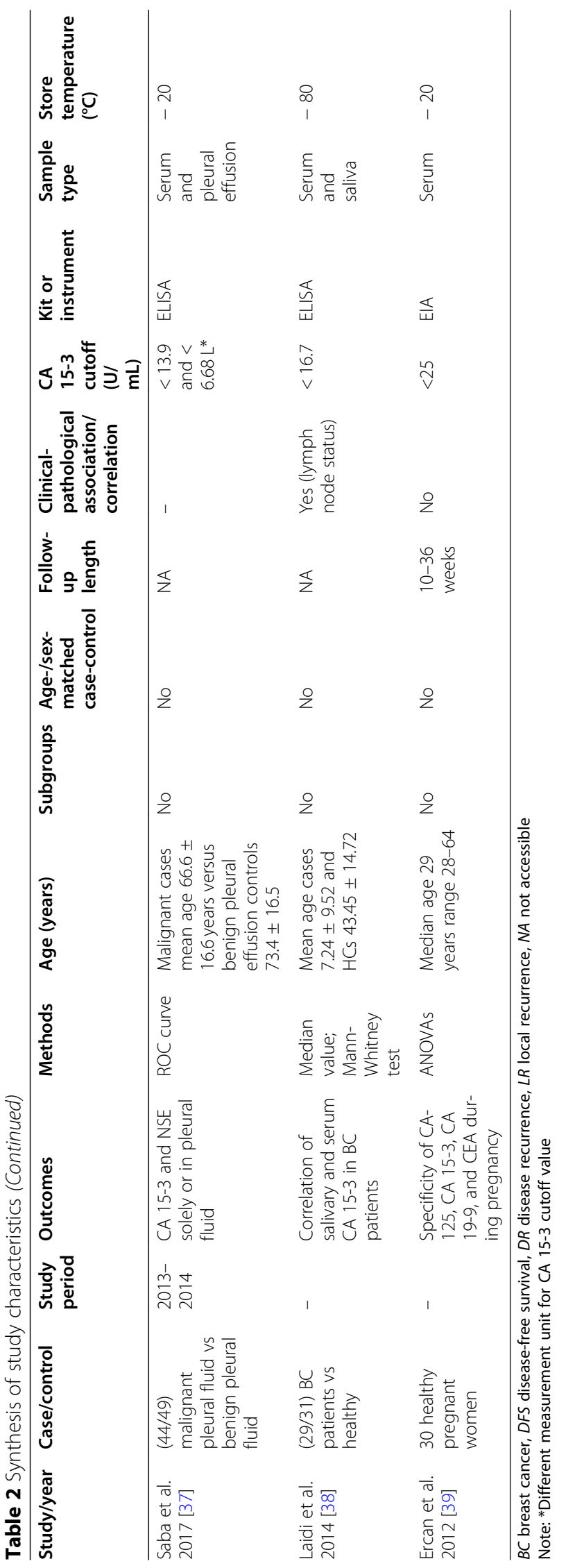




\section{Supplementary Information}

The online version contains supplementary material available at https://doi. org/10.1186/s13643-021-01631-7.

Additional file 1. Preferred Reporting Items for Systematic Reviews and Meta-Analyses (PRISMA) standards and guidelines.

Additional file 2. New Castle Ottawa Scale (NOS) for case-control study quality assessment.

\section{Acknowledgements}

None.

\section{Authors' contributions}

LC wrote and reviewed the manuscript, conceived the figures, and performed a bibliographic search. AC reviewed the manuscript and participated in the bibliographic search. KP and MF contributed to the manuscript review and data analyses. PM conceived the work, wrote and reviewed the manuscript, and participated in the bibliographic search. MS coordinated the activity of all participants, revised the manuscript, and gave final approval. All authors have read and agreed to the published version of the manuscript

\section{Funding}

This work was supported by "Progetti di Ricerca Corrente," funded by the Italian Ministry of Health.

\section{Declarations}

\section{Ethics approval and consent to participate}

Not applicable.

\section{Consent for publication}

All authors approved the final version of the manuscript and agreed to its submission for publication.

\section{Competing interests}

The authors declare that they have no competing interests.

Received: 15 October 2020 Accepted: 10 March 2021

Published online: 09 April 2021

\section{References}

1. Kamtchum-Tatuene J, Jickling GC. Blood biomarkers for stroke diagnosis and management. Neuromolecular Med. 2019;21(4):344-68. https://doi. org/10.1007/s12017-019-08530-0.

2. Kilgour E, Rothwell DG, Brady G, Dive C. Liquid biopsy-based biomarkers of treatment response and resistance. Cancer Cell. 2020;37(4):485-95. https:// doi.org/10.1016/j.ccell.2020.03.012

3. Smaldone $\mathrm{G}$, Beneduce $\mathrm{G}$, Incoronato M, Pane $\mathrm{K}$, Franzese M, Coppola L et al. KCTD15 is overexpressed in human childhood B-cell acute lymphoid leukemia. Sci Rep. 2019;9(1):20108. https://doi.org/10.1038/s41598-01956701-7.

4. Pane K, Mirabelli P, Coppola L, Illiano E, Salvatore M, Franzese M. New roadmaps for non-muscle-invasive bladder cancer with unfavorable prognosis. Front Chem. 2020;8. https://doi.org/10.3389/fchem.2020.00600.

5. Coppola L, Cianflone A, Grimaldi AM, Incoronato M, Bevilacqua P, Messina F, et al. Biobanking in health care: evolution and future directions. J Transl Med. 2019:17(1):172. https://doi.org/10.1186/s12967-019-1922-3.

6. Hilkens J, Vos HL, Wesseling J, Peterse J, Storm J, Boer M, et al. The expression levels of episialin in human carcinomas are sufficiently high to potentially interfere with adhesion and promote metastasis. Breast Cancer Adv Biol Ther. 1996:281-288.

7. Mukhopadhyay P, Chakraborty S, Ponnusamy MP, Lakshmanan I, Jain M, Batra SK. Mucins in the pathogenesis of breast cancer: implications in diagnosis, prognosis and therapy. Biochim Biophys Acta Rev Cancer. 2011; 1815(2):224-40. https://doi.org/10.1016/j.bbcan.2011.01.001.

8. Li JX, Liu LY, Feng ZW, Wang X, Huang YB, Dai HJ, et al. Tumor markers CA15-3, CA125, CEA and breast cancer survival by molecular subtype: a cohort study. Breast Cancer. 2020;27(4):621-30. https://doi.org/10.1007/s122 82-020-01058-3.
9. Le Rhun E, Kramar A, Salingue S, Girot M, Rodrigues I, Mailliez A, et al. CSF CA 15-3 in breast cancer-related leptomeningeal metastases. J Neurooncol. 2014;117(1):117-24. https://doi.org/10.1007/s11060-014-1361-1.

10. Sturgeon CM, Duffy MJ, Stenman UH, Lilja H, Brunner N, Chan DW, et al. National Academy of Clinical Biochemistry Laboratory Medicine Practice Guidelines for use of tumor markers in testicular, prostate, colorectal, breast, and ovarian cancers. Clin Chem. 2008;54(12):E11-79. https://doi.org/10.1373/ clinchem.2008.105601.

11. Shao YB, Sun XF, He YN, Liu CJ, Liu H. Elevated levels of serum tumor markers CEA and CA15-3 are prognostic parameters for different molecular subtypes of breast cancer. PLoS One. 2015;10(7). https://doi.org/10.1371/ journal.pone.0133830.

12. Wu DW, Li YM, Wang F. How long can we store blood samples: a systematic review and meta-analysis. Ebiomedicine. 2017;24:277-85. https:// doi.org/10.1016/j.ebiom.2017.09.024

13. Gunawardena D, Jayaweera S, Madhubhashini G, Lokumarakkala DD, Senanayake SJ. Reliability of parameters of complete blood count with different storage conditions. J Clin Lab Anal. 2017;31(2).

14. Hanon EA, Sturgeon CM, Lamb EJ, PTH ISDWG. Sampling and storage conditions influencing the measurement of parathyroid hormone in blood samples: a systematic review. Clin Chem Lab Med. 2013;51(10):1925-41. https://doi.org/10.1515/cclm-2013-0315.

15. Bonilla-Sepúlveda ÓA. Tumor markers in breast cancer. Systematic review. Ginecol Obstet Méx. 2020;88(12):860-9.

16. Fu YJ, Li H. Assessing clinical significance of serum CA15-3 and carcinoembryonic antigen (CEA) levels in breast cancer patients: a metaanalysis. Med Sci Monit. 2016;22:3154-62. https://doi.org/10.12659/MSM. 896563.

17. Wu Q, Li M, Zhang S, Chen L, Gu X, Xu F. Clinical diagnostic utility of CA 153 for the diagnosis of malignant pleural effusion: a meta-analysis. Exp Ther Med. 2015;9(1):232-8. https://doi.org/10.3892/etm.2014.2039.

18. Moher D, Liberati A, Tetzlaff J, Altman DG, Grp P. Preferred reporting items for systematic reviews and meta-analyses: the PRISMA statement. PLoS Med. 2009:6(7)

19. Siravegna G, Marsoni S, Siena S, Bardelli A. Integrating liquid biopsies into the management of cancer. Nat Rev Clin Oncol. 2017;14(9):531-48. https:// doi.org/10.1038/nrclinonc.2017.14.

20. Spoorenberg SMC, Vestjens SMT, Voorn GP, van Moorsel CHM, Meek B, Zanen $P$, et al. Course of SP-D, YKL-40, CCL18 and CA 15-3 in adult patients hospitalised with community-acquired pneumonia and their association with disease severity and aetiology: a post-hoc analysis. PLoS One. 2018;13(1).

21. Wells G, Shea B, O'Connell D, Peterson J, Welch V, Losos M, Tugwell P. The Newcastle-Ottawa Scale (NOS) for assessing the quality of nonrandomised studies in meta-analyses. 2013. http://www.ohri.ca/programs/clinical_ epidemiology/oxford.asp.

22. Chukwurah E, Emele F, lyare F. Breast cancer in Nigerian women: evaluating the utility of circulating immune complexes and cancer antigens (CA 15-3 and CA 27.29) in disease surveillance. Res J Immunol. 2018;11:7-14.

23. Mahmood M, Haq MF, Nizami NA, Akram S, Choudhry N, Yasmin R. Comparison of serum ferritin with carbohydrate antigen 15-3 (CA 15-3) in breast cancer patients in Pakistan. Pak J Med Health Sci. 2016;10(4):1384-9.

24. Said NM. Three gold indicators for breast cancer prognosis: a case-control study with ROC analysis for novel ratios related to CBC with (ALP and LDH). Mol Biol Rep. 2019;46(2):2013-27. https://doi.org/10.1007/s11033-019-04650-9.

25. Moazzezy N, Farahany TZ, Oloomi M, Bouzari S. Relationship between preoperative serum CA15-3 and CEA levels and clinicopathological parameters in breast cancer. Asian Pac J Cancer Prev. 2014;15(4):1685-8. https://doi.org/10.7314/APJCP.2014.15.4.1685.

26. El-Moneim Ebied SA, El-Moneim NAA, Hewala TL, Anwar MM, Rabi SM. The diagnostic, prognostic and follow-up value of serum $\mathrm{BCl}-2$, Bax and p53 proteins in breast cancer patients: a comparison with serum CA 15-3. Middle East J Cancer. 2013:4(2):51-62.

27. Svobodova S, Kucera R, Fiala O, Karlikova M, Narsanska A, Zednikova I, et al. CEA, CA 15-3, and TPS as prognostic factors in the follow-up monitoring of patients after radical surgery for breast cancer. Anticancer Res. 2018;38(1): 465-9. https://doi.org/10.21873/anticanres.12245.

28. Pedersen AC, Sorensen PD, Jacobsen EH, Madsen JS, Brandslund I. Sensitivity of CA 15-3, CEA and serum HER2 in the early detection of recurrence of breast cancer. Clin Chem Lab Med. 2013;51(7):1511-9. https:// doi.org/10.1515/cclm-2012-0488. 
29. Di Gioia D, Dresse M, Mayr D, Nagel D, Heinemann V, Stieber P. Serum HER2 in combination with CA 15-3 as a parameter for prognosis in patients with early breast cancer. Clin Chim Acta. 2015;440:16-22. https://doi.org/10.1016/ j.cca.2014.11.001

30. Sen S, Kuru O, Akbayir O, Oguz H, Yasasever V, Berkman S. Determination of serum CRP, VEGF, Leptin, CK-MB, CA-15-3 and IL-6 levels for malignancy prediction in adnexal masses. J Turk Ger Gynecol Assoc. 2011;12(4):214-9. https://doi.org/10.5152/jtgga.2011.54.

31. Metwally FM, El-mezayen HA, Ahmed HH. Significance of vascular endothelial growth factor, interleukin-18 and nitric oxide in patients with breast cancer: correlation with carbohydrate antigen 15.3. Med Oncol. 2011; 28:S15-21. https://doi.org/10.1007/s12032-010-9657-2.

32. Hewala Tl, Abd El-Monaim NA, Anwar M, Ebied SA. The clinical significance of serum soluble Fas and p53 protein in breast cancer patients: comparison with serum CA 15-3. Pathol Oncol Res. 2012;18(4):841-8. https://doi.org/10.1 007/s12253-012-9512-1.

33. Zajkowska M, Gacuta E, Lubowicka E, Szmitkowski M, Lawicki S. Can VEGFR3 be a better tumor marker for breast cancer than CA 15-3? Acta Biochim Pol. 2020;67(1):25-9. https://doi.org/10.18388/abp.2020_2873.

34. Zaleski M, Kobilay M, Schroeder L, Debald M, Semaan A, Hettwer K, et al. Improved sensitivity for detection of breast cancer by combination of miR34a and tumor markers CA 15-3 or CEA. Oncotarget. 2018;9(32):22523-36. https://doi.org/10.18632/oncotarget.25077.

35. Tang Z, Li L, Shen L, Shen X, Ju S, Cong H. Diagnostic value of serum concentration and integrity of circulating cell-free DNA in breast cancer: a comparative study with CEA and CA 15-3. Lab Med. 2018;49(4):323-8. https://doi.org/10.1093/labmed/lmy019.

36. Christenson RH, Cervelli DR, Sterner J, Bachmann LM, Rebuck H, Gray J, et al. Analytical performance and clinical concordance of the cancer biomarkers CA 15-3, CA 19-9, CA 125 II, carcinoembryonic antigen, and alphafetoprotein on the Dimension Vista (R) System. Clin Biochem. 2011;44(13): 1128-36. https://doi.org/10.1016/j.clinbiochem.2011.06.009.

37. Saba MA, Valeh T, Ehteram H, Haddad Kashani H, Ghazi ZM. Diagnostic value of neuron-specific enolase (NSE) and cancer antigen 15-3 (CA 15-3) in the diagnosis of pleural effusions. Asian Pac J Cancer Prev. 2017;18(1):257-61.

38. Laidi F, Bouziane A, Lakhdar A, Khabouze S, Amrani M, Rhrab B, et al. Significant correlation between salivary and serum Ca 15-3 in healthy women and breast cancer patients. Asian Pac J Cancer Prev. 2014;15(11): 4659-62. https://doi.org/10.7314/APJCP.2014.15.11.4659.

39. Ercan S, Kaymaz O, Yucel N, Orcun A. Serum concentrations of CA 125, CA 153, CA 19-9 and CEA in normal pregnancy: a longitudinal study. Arch Gynecol Obstet. 2012;285(3):579-84. https://doi.org/10.1007/s00404-011-2025-4.

40. Flores CFY, de Las Mercedes Hurtado Pineda A, Bonilla VMC, Saenz-Flor K. Sample management: stability of plasma and serum on different storage conditions. Ejifcc. 2020;31(1):46-55.

41. Cuhadar S, Koseoglu M, Atay A, Dirican A. The effect of storage time and freeze-thaw cycles on the stability of serum samples. Biochem Med. 2013; 23(1):70-7. https://doi.org/10.11613/bm.2013.009.

42. Hewitt R, Watson P. Defining biobank. Biopreserv Biobank. 2013;11(5):30915. https://doi.org/10.1089/bio.2013.0042.

43. Hartman V, Castillo-Pelayo T, Babinszky S, Dee S, Leblanc J, Matzke L, et al. Is your biobank up to standards? A review of the National Canadian Tissue Repository Network required operational practice standards and the controlled documents of a certified biobank. Biopreserv Biobank. 2018;16(1): 36-41. https://doi.org/10.1089/bio.2017.0065

44. Schenk M, Huppertz B, Obermayer-Pietsch B, Kastelic D, Hormann-Kropfl M, Weiss G. Biobanking of different body fluids within the frame of IVF-a standard operating procedure to improve reproductive biology research. J Assist Reprod Genet. 2017;34(2):283-90. https://doi.org/10.1007/s10815-0160847-5.

\section{Publisher's Note}

Springer Nature remains neutral with regard to jurisdictional claims in published maps and institutional affiliations.

Ready to submit your research? Choose BMC and benefit from:

- fast, convenient online submission

- thorough peer review by experienced researchers in your field

- rapid publication on acceptance

- support for research data, including large and complex data types

- gold Open Access which fosters wider collaboration and increased citations

- maximum visibility for your research: over $100 \mathrm{M}$ website views per year

At BMC, research is always in progress.

Learn more biomedcentral.com/submissions 